\title{
Stanisław Liszewski
}

\section{L'ENSEIGNEMENT DANS LE DOMAINE DU TOURISME A L'UNIVERSITÉ DE モÓDŹ}

\author{
KSZTAECENIE W ZAKRESIE TURYZMU \\ NA UNIWERSYTECIE ŁÓDZKIM
}

Dans l'article, on a présenté l'idée d'enseigner le géographe du tourisme à l'Université de Łódź ainsi que cinq blocs des matières constituant le programme de l'enseignement et la silhouette d'un diplômé de la spécialisation discutée. L'article est complété par le plan détaillé des études de la géographie du tourisme, la liste des manuels et des abrégés recommandés aux étudiants de cette spécialisation.

L'histoire de l'enseignement des spécialistes pour les besoins du tourisme se développant sans cesse est, à l'Université de Eódź, courte et, quant à l'organisation, dès le commencement liée à la direction géographique. Il est vrai que, dans le passé, on essayait d'enseigner individuellement l'économie du tourisme: quelques travaux particuliers ont été préparés à la section de l'économie du commerce à la Faculté d'Economie et Sociologie; ces essais n'ont pas trouvé leur expression formelle dans la spécialisation ou la direction de l'enseignement.

Pour le début de l'enseignement universitaire des spécialistes pour les besoins du tourisme à Łódź, il faut reconnaître l'ouverture en 1977 du Centre Postdiplômier du Tourisme auprès de l'ancien Institut de Géographie. Ce centre agit avec succès jusqu'à nos jours auprès de l'Institut de Géographie Economique et d'Organisation de l'Espace. Il instruit les spécialistes possédant les diplômes de diverses écoles supérieures, travaillant professionnellement ou en activistes sociaux dans l'économie touristique largement comprise. Jusqu'à la fin de l'année 1988191 personnes venant de toutes les régions de la Pologne ont obtenu leur diplôme de fin d'études au Centre Postdiplômier du Tourisme, qui a le caractère de perfectionnement et de complètement du savoir specialiste indispensable à tous ceux qui travaillent dans le tourisme. 
Il est à noter que le programme du Centre a le caractère interdisciplinaire et sa réalisation dure deux semestres.

Les études universitaires de base dans le domaine du tourisme ont été initiées à l'Université de Eódź en octobre 1982. C'est alors qu'on a créé la spécialisation dans la sphère de la géographie du tourisme à la section géographique de la Faculté de Biologie et de Sciences de la Terre. Cette spécialisation commence après la deuxième année du cours général des études géographiques, dure trois années et finit par la présentation d'un travail de maîtrise et l'examen après la Vème année. C'est alors qu'on obtient la licence en géographie avec la spécialisation de géographie du tourisme. Jusqu'à l'an académique 1988/1989 70 étudiants ont entrepris les études spécialistes; 39 d'entre eux ont obtenu leur diplôme jusqu'à la fin de l'année 1988, les autres continuent leurs études. Il faut signaler que le recrutement est limité '(12 étudians par an) et le nombre de personnes voulant entreprendre ces études dépasse les limites fixées.

L'ouverture de la spécialisation dans le domaine de la géographie du tourisme à l'Université de Łódź était précédée d'une large discussion tendant à la détermination de l'idée de cette spécialisation, du profil de l'enseignement et de la silhouette du futur diplômé, surtout de sa préparation au travail professionnel. L'expérience acquise pendant l'organisation et ensuite la réalisation du programme au Centre Postdiplômier du Tourisme a facilité ces discussions.

L'instruction universitaire d'un géographe embrasse traditionnellement le savoir naturel, social et économique qui permet de mieux comprendre le monde nous entourant et les processus y survenant. Les connaissances acquises dans le cadre de l'instruction géographique font que le géographe peut être reconnu pour un porteur du savoir, des idées humanistes et naturelles dans le tourisme. Le géographe doté d'une possibilité de voir la réalité synthétiquement et largement est préparé à remplir cette partie du temps libre que l'homme réserve au tourisme et au repos.

L'idée de l'instruction d'un géographe du tourisme au centre de Łódź prévoit la préparation d'un tel diplômé qui saurait remplir du contenu essentiellement cognitif les formes diverses d'organisation du tourisme et du repos. Ce sera donc un organisateur, conscient de ses objectifs, du temps libre dans le domaine de sa disposition active, cognitive et touristique. Le propagateur et la réalisateur du tourisme en liaison avec les éléments cognitifs, il exposera, en même temps, l'étroite union avec la nature. Ce doit être un homme au savoir professionnel approfondi mais aussi un activiste social.

L'idée supposée de la silhouette du géographe du tourisme exigeait 
la concrétisation des matières de programmes de l'instruction du diplômé futur.

Les principes du programme de la spécialisation dans la géographie du tourisme sont réalisés en III ème, IVème et Vème années de géographie et comprennent ensemble 870 heures de conférences et 63 jours (462 heures) de pratiques professionnelles et d'exercices sur le terrain (Tabl. I). Ici il faut tenir compte du fait que le programme de cinq ans d'études géographiques embrasse au total 3780 heures de conférences, dont $23 \%$, donc un quart à peu près est destiné aux activités spécialistes. Dans la suite de cet article, nous ne nous occuperons que de ces activités; cependant il faut se souvenir de l'influence qu'exercent sur l'exécution de la profession future les autres matières géographiques.

Tableau I

Le plan des études de la géographie du tourisme à l'Université de Łódź

Le nom de matière L'année Le nombre d'heures des activités des études au total conférences exercices

Examen

des études au total conférences exercices

Les bases de la géogra-

phie du tourisme

III

60

30

30

E

La physiologie du travail et repos avec les éléments de l'hygiène

Le proséminaire spécialiste

III

30

15

60

-

15

III

Les problemes choisis de l'architecture et de l'art

60

60

Les méthodes de recherches dans la géographie du tourisme

a géographie touristique de Pologne

Le milieu naturel dans le tourisme

La matière facultative (langue étrangère)

L'économie du tourisme avec l'organisation et la technique du service des entreprises touristiques

L'aménagement touristique

La chorographie

La géographie touristique du monde

$\begin{array}{ll}- & 60 \\ 30 & 30 \\ 15 & 30 \\ 30 & 30\end{array}$


Tableau I (suite)

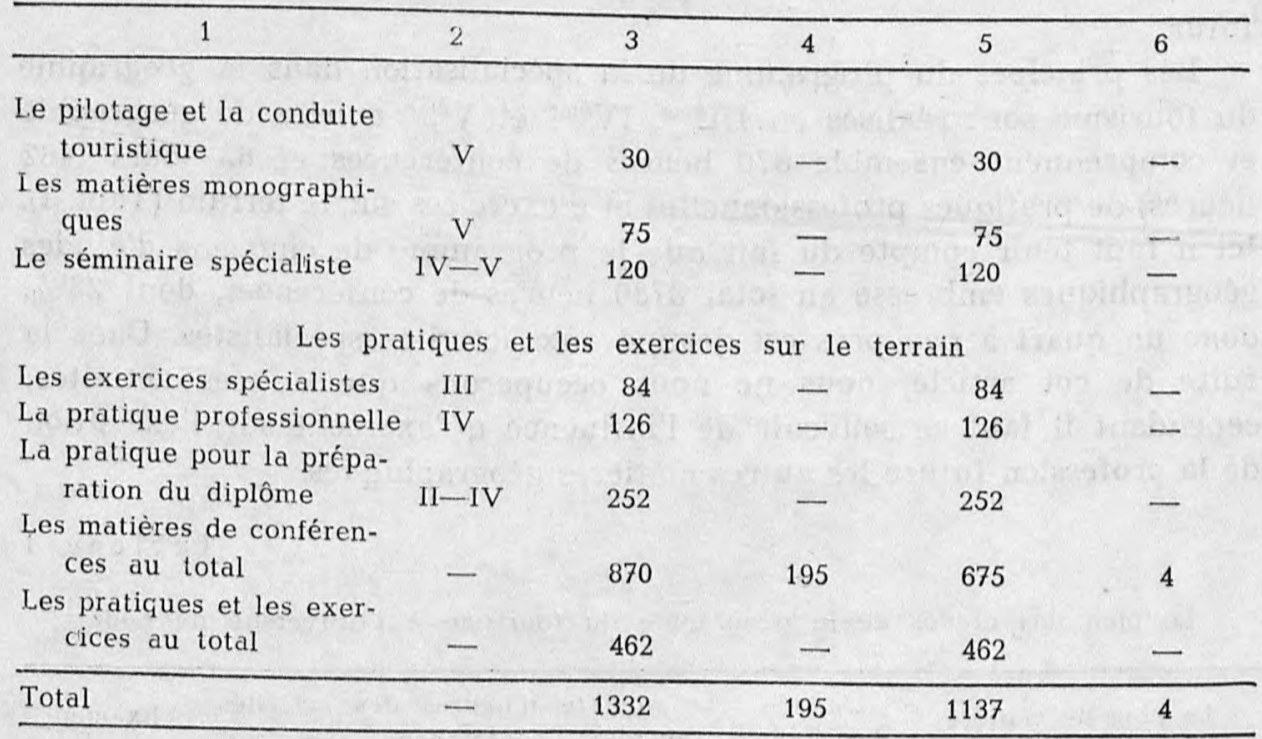

Dans le cadre du programme de spécialisation dans la géographie du tourisme, les étudiants participent aux conférences concernant $15 \mathrm{ma}$ tières, dont 4 aboutissent à un examen, et sont obligés de faire trois genres de pratiques et d'exercices sur le terrain.

Le programme de la spécialisation dans la géographie du tourisme réalisé à l'Université de Łódź embrasse les matières qu'on pourrait lier en cinq blocs compacts (Tabl. II).

Tableau il

Les blocs thématiques de l'enseignement des étudiants

se spécialısant dans la géographie du tourisme à l'Université de Łódź

\begin{tabular}{lcccccr}
\hline \multirow{2}{*}{ Nom du bloc } & Conférences & $\begin{array}{c}\text { Pratiques } \\
\text { et exercices } \\
\text { sur le terrain }\end{array}$ & Activités au total \\
\cline { 2 - 7 } & $\begin{array}{c}\text { nombre } \\
\text { d'heures }\end{array}$ & $\%$ & $\begin{array}{c}\text { nombre } \\
\text { d'heures }\end{array}$ & $\%$ & $\begin{array}{r}\text { nombre } \\
\text { d'heures }\end{array}$ & $\%$ \\
\hline $\begin{array}{l}\text { L'homme en tant que participant } \\
\quad \text { du mouvement touristique }\end{array}$ & 30 & 3,6 & - & - & 30 & 2,3 \\
Les ressources touristiques & 180 & 20,6 & - & - & 180 & 13,5 \\
La géographie touristique & 180 & 20,6 & - & - & 180 & 13,5 \\
Le bloc professionnel et pratique & 240 & 27,6 & 126 & 27,3 & 366 & 27,5 \\
Le bloc théorique et méthodique & 240 & 27,6 & 336 & 72,7 & 576 & 43,2 \\
\hline Total & 870 & 100,0 & 462 & 100,0 & 1332 & 100,0 \\
\hline
\end{tabular}


Le premier de ces blocs comprend les matières dont le but est la connaissance de l'homme en tant que participant du mouvement touristique, de ses besoins et possibilités physiques et psychiques. C'est l'élément indispensable de l'instruction rendant possible la compréhension des individus et des groupes sociaux et professionnels avec lesquels le diplômé futur de la géographie du tourisme sera obligé de travailler. La connaissance de la physiologie de travail et de repos, ainsi que des éléments de la psychologie sociale et de la sociologie doit faciliter, à l'organisateur potentiel du temps libre, le choix d'une forme convenable du repos actif ou bien celui des formes convenables d'organisation, selon les états ou les comportements psychiques considérés comme probables.

Le deuxième bloc thématique a été destiné à la présentation des ressources touristiques, ainsi naturelles que anthropogéniques. Le savoir sur la diversité des valeurs touristiques est un élément indispensable de l'instruction d'un géographe qui doit réaliser les idées de l'organisation méritoire du temps libre. L'étendue du savoir sur les ressources touristiques de celui qui étudie la géographie du tourisme est large: à côté des valeurs du milieu naturel, y compris les terrains protégés par la loi, elle embrasse l'art, l'architecture, l'ethnographie, aussi bien la ville, présentée d'une façon complexe, et l'établissement industriel en tant qu'un objet touristique. Le savoir que donne cette partie des études doit constituer une portion considérable de la charge méritoire de l'organisateur futur du temps libre, lui rendant la possibilité de présenter convenablement les offres touristiques.

Le caractère du troisième bloc des matières, qui embrasse les géographiques et touristiques présentant les phénomènes et les processus conçus comme spatiaux, est analogue à celui du précédent. Le savoir conquis pendant l'étude de la géographie du tourisme, de la géographie touristique de la Pologne, ou de celle touristique du monde approfondit les connaissances sur la disposition des objets, des centres et des régions - terrains potentiels du mouvement touristique.

Le quatrième bloc touristique, englobe le savoir professionnel et pratique préparant dans le domaine de l'organisation et de la pratique du travail dans le tourisme. La sphère des matières de ce bloc est hétérogène et comprend l'économie du tourisme, la chorographie, l'aménagement touristique, ainsi que l'organisation et la technique du service des entreprises, la surveillance et l'accompagnement des touristes. Ce bloc est complété par cette langue étrangère, que l'étudiant apprenait pendant ses premières trois annés d'études, et par les activités pratiques professionnelles que les étudiants exécutent dans les bureaux et les institutions touristiques pendant trois semaines. Ce large éventail des 
matières permet au géographe de faire connaissance des problèmes et de la spécificité des postes de travail différents dans le domaine du tourisme et du repos. Ces éléments du savoir professionnel, dans lesquels le rôle du géographe peut accomplir la fonction primordiale, sont dignes d'être soulignés d'une façon particulière. Cela se rapporte avant tout à l'organisation des entreprises, à la surveillance et l'accompagnement des touristes, l'aménagement touristique et la chorographie.

Le dernier, cinquième bloc thématique groupe les matières constituant le compendium du savoir théorique et méthodique. Ce sont le savoir et les connaissances indispensables pour la préparation d'un travail scientifique autonome, qu'est la maîtrise, mais aussi très utiles dans le travail professionnel futur, surtout pour faire les analyses et les études, pour savoir exploiter l'acquis scientifique accumulé dans ce domaine des connaissances. L' étudiant se prépare au travail scientifique en participant aux proséminaires et séminaires de maîtrise; quelques matières l'introduisent dans les problèmes des méthodes et des techniques de recherches. Il s'y prépare aussi pendant les exercices spécialistes sur le terrain et les pratiques destinées à l'accumulation des matières utiles pour son propre travail.

La division du temps destiné aux activités dans les blocs thématiques particuliers n'est pas uniforme; elle accentue le caractère de l'instruction du futur diplômé. Diverse est aussi la participation des blocs dans les conférences, les pratiques et les exercices sur le terrain. Quant aux conférences, les activités au caractère théorique et méthodique et celles au caractère professionnel et pratique prennent le même espace de temps $(27,6 \%)$. Un peu moindre est la participation des matières se rapportant aux ressources touristiques, celles géographiques et touristiques; cependant dans les deux cas elle ne diffère pas et égale $20,6 \%$ de toutes activités au caractére de conférence. Le moins de temps prennent dans les activités spécialistes les matières présentant l'homme et ses besoins $(3,6 \%)$. Pourtant, il faut le dire, les étudiants en géographie, dans le cadre des matières générales, obtiennent une grande portion de savoir du domaine de la psychologie, de la sociologie et de la pédagogie.

Le pourcentage de participation des blocs particuliers change, si l'on prend en considération les pratiques et les exercices sur le terrain. Ces activités enrichissent d'une façon inégale seulement deux blocs thématiques; il s'ensuit qu'au bilan total des activités à la spécialisation: géographie du tourisme, à l'Université de Łódź, domine le bloc des matières théoriques et méthodiques (Tabl. II).

La division et la structure de la matière d'enseignement de la spécialisation présentée facilite la compréhension de la conception 
générale de l'instruction dans le domaine de la géographie du tourisme à l'Université de Eódż.

Elle conçoit que le diplômé, ayant fait ses études spécialistes, doit:

A - connaitre l'objet de sa future activité professionnelle, donc l'homme voulant pratiquer le tourisme, ses possibilités et besoins, aussi physiques que psychiques;

B - connaître les ressources touristiques avec leur spécificité et différenciation régionale, aussi bien en Pologne que dans le monde; cette connaissance doit être telle qu'on puisse proposer d'une façon responsable les formes et les lieux touristiques les plus convenables à un homme concret;

C - savoir organiser et servir une entreprise touristique, aménager le temps libre du touriste d'une façon conforme à ses possibilités et ses intérêts;

D - savoir pratiquement utiliser l'acquis scientifique pour les besoins de la profession exécutée, approfondir et perfectionner ses connaissances dans la discipline tellement étendue, qu'est le tourisme.

L'idée d'enseignement que je viens de présenter et le niveau de la spécialisation en géographie du tourisme réaiisée à l'Université de Łódź conçoivent la préparation des spécialistes, dont les connaissances prédestinent à la programmation du repos actif ainsi des touristes individuels que des groupes ou ensembles de personnes. Les géographes diplômés, spécialistes du tourisme, peuvent être organisateurs et animateurs du mouvement touristique et du repos dans les établissements (travailleurs des services sociaux sur le plan touristique), les organisations sociales au divers profil d'activité (Société Touristique et Chorographique Polonaise), organisations de la jeunesse, etc. Ils peuvent aussi utiliser leur savoir en travaillant dans les agences de tourisme et de repos, où leur acquis peut être utilisé pour la programmation et l'organisation des entreprises chorographiques et du repos actif. Leur travail avec la jeunesse, aussi bien dans les écoles que d'autres organisations d'instruction publique, peut être couronné de succès. En s'occupant de pédagogie, ils peuvent en même temps devenir organisateurs du mouvement touristique et du repos actif. Leurs connaissances peuvent et doivent être exploitées aussi dans les travaux concernant la programmation dans les maisons de repos et les entreprises.

L'idée d'enseignement dans le cadre de la spécialisation: géographie du tourisme à l'Université de Łódź conçoit aussi la préparation du diplômé à savoir prévoir et indiquer ces éléments de l'aménagement matériel de l'espace, qui doivent servir l'organisation correcte du temps libre. Ces connaissances et avant tout le savoir acquis font que le 
diplômé, spécialiste de la géographie du tourisme, peut entreprendre le travail dans les bureaux et les institutions s'occupant de planification spatiale à l'échelle diverse, et dans les établissements décidant du mouvement du tourisme et du repos.

Le caractère et la spécificité des études universitaires dans le domaine de la géographie du tourisme se font voir particulièrement intensement dans la thématique des travaux de maitrise préparés dans le Centre de Łódź. Dans leurs premières élaborations scientifiques, les jeunes gens exploitent leurs propres et originaux matériaux accumulés pendant les recherches sur le terrain. Les problèmes, souvent menus et contributifs qui y sont résolus, démontrent le sens et la nécessité des études scientifiques, éveillent l'intérêt du jeune chercheur, lui font apprendre l'honnêteté de recherches scientifiques et, avant tout découvrent l'immensité des problèmes liés au tourisme et au repos dans notre pays.

La thématique des travaux de maîtrise réalisés dans le cadre de la spécialisation se lie au profil des recherches de l'Institut de la Géographie des villes et du Tourisme, dont cette spécialisation fait partie et aux intérêts individuels des étudiants. Les travaux entrepris concernent la problématique:

- de la colonisation touristique, de sa génèse et des changements qui s'y opèrent sous l'influence du mouvement touristique;

- de la fonction touristique et de repos des localités, terrains et régions;

- de l'urbanisation des terrains ruraux sous l'influence du tourisme et du repos;

- des valeurs ou ressources touristiques des objets et terrains;

- de l'influence du tourisme sur les transformations du milieu naturel;

- du repos des habitants des villes aux grandeurs et fonctions différentes.

La dernière thématique était réalisée dans plusieurs travaux de maîtrise dont le but consistait en essai de construire les modèles spaciaux du repos des habitants des villes.

Les conceptions idéales et le programme de la spécialisation dans la géographie du tourisme à l'Université de Eódź visent à la préparation d'un diplômé, auquel les principes et les bases de l'économie dite touristique, dont en Pologne s'occupent avant tout les économistes, ne soient pas étrangers; ses connaissances doivent remplir cette économie de sujets méritoires profondement humanistes. Il s'agit surtout de l'aménagement du temps libre, dont l'étendue grandit toujours dans notre pays. 
La réalisation du programme didactique ainsi conçu rencontre des barrières et des obstacles qui résultent du fait l'histoire de l'éducation formalisée des spécialistes pour les besoins du tourisme n'est pas longue, son expérience et son acquis didactique sont bornés. Cela se fait voir surtout dans le choix très limité des manuels et des abrégés, utilisés dans le processus didactique.

Le caractère experimental des études spécialistes de la géographie $\mathrm{du}$ tourisme à l'Université de Éodź oblige à l'investigation sur les programmes didactiques des autres écoles supérieures en Pologne et à l'étranger et à la confrontation de ceux-ci avec le programme réalisé chez nous. Ces confrontations doivent contribuer au perfectionnement de la formation des cadres nouveaux et à l'adaptation de cette formation aux besoins de l'économie touristique se développant sans cesse.

Liste des manuels recommandés aux étudiants de géographie du tourisme à l'Université de Łódź

1. Bartkowski T., 1977, Wypisy do geografii turyzmu, cz. 1, AWF Poznań, skrypt nr 111.

2. Deja W., 1985, Przewodnik do ćwiczeń z geografii turystycznej, AWF Poznań, skrypt nr 113.

3. Dubaniewicz H., Nurek T., Zawadzka A., 1988, Biometeorologia turystyki. Wybrane zagadnienia $z$ biometeorologii człowieka, cz.1, AWF Gdańsk.

4. Gaworecki W. W., 1982, Ekonomika i organizacja turystyki, PWN, Warszawa.

5. Kornak A., 1979, Ekonomika turystyki, PWN, Warszawa.

6. Kruczek Z., 1979, Metodyka krajoznawstwa, AWF Kraków, Wydawnictwa skryptowe $\mathrm{nr} 37$.

7. Kruczek Z., Sacha S., 1983, Geografia walorów turystycznych Polski, AWF Kraków, Wydawnictwa skryptowe $\mathrm{nr} 63$.

8. Lijewski T., Mikułowski B., Wyrzykowski J., 1985, Geografia turystyki Polski, PWE,Warszawa.

9. Lipniacki W., 1986, Elementy teorii krajoznawstwa, t. 2, PTTK, Szczecin.

10. Praca zbiorowa pod red. J. Warszyńskiej, 1988, Regionalna geografia turystyczna świata, cz. 2, Kraje pozaeuropejskie, UJ., Skrypty uczelniane nr 578, Kraków.

11. Sacha S., 1981, Geografia turystyczna krajów europejskich, AWF Kraków, Wydawnictwa skryptowe $\mathrm{nr} 42$.

12. Rogalewski O., 1979, Zagospodarowanie turystyczne, WSiP, Warszawa. 


\section{Warszyńska J., Jackowski A., 1978, Podstawy geografii turyzmu, PWN, Warszawa.}

prof. dr hab. Stanisław Liszewski

Wpłynęło:

Zakład Geografii Miast i Turyzmu

15 grudnia 1989

Instytutu Geografii Ekonomicznej

i Organizacji Przestrzeni

Uniwersytetu Łódzkiego

Al. Kościuszki 21

90-418 Łódż (Pologne)

Traduit par Lucjan Kowalski

\section{STRESZCZENIE}

Kształcenie specjalistow na potrzeby turystyki łączy się na Uniwersytecie Łódzkim z kierunkiem geograficznym, na którym w 1977 r. uruchomiono najpierw Podyplomowe Studium Turystyki, a od $1982 \mathrm{r}$. specjalizację z geografii turyzmu.

Idea wykształcenia geografa turyzmu w ośrodku łódzkim zakłada przygotowanie takiego absolwenta, który będzie posiadał umiejętności nasycenia treścią, głównie poznawczą, różnorodnych form turystyki i wypoczynku. Ma to być świadomy celów organizator czasu wolnego w zakresie czynnego, poznawczo-turystycznego jego zagospodarowania.

Założenia programowe specjalizacji z geografii turyzmu realizowane są na III, IV i $V$ roku kierunku geograficznego i obejmują 870 godzin zajęć audytoryjnych oraz 63 dni praktyk zawodowych i ćwiczeń terenowych (tab. I).

Program zajęć specjalizacyjnych można podzielić na pięć bloków tematycznych (tab. II). Podział czasu przeznaczonego na zajęcia w poszczególnych blokach nie jest jednakowy i podkreśla charakter wykształcenia przyszłego absolwenta kończącego studia uniwersyteckie. Różny jest też udział poszczególnych bloków w zajęciach audytoryjnych oraz praktykach i ćwiczeniach terenowych.

Realizowany program dydaktyczny zakłada, iż absolwent specjalizacji powinien:

A - znać możliwości i potrzeby, tak fizyczne, jak i psychiczne czlowieka pragnącego uprawiać turystykęę;

B - znać zasoby turystyczne Polski i świata na tyle, aby móc w sposób odpowiedzialny proponować najwłaściwsze dla konkretnego człowieka formy i miejsca uprawiania turystyki;

C - umieć zorganizować i obsłużyć imprezę turystyczną, a także zagospodarować czas wolny turysty;

D - umieć praktycznie zużytkować dorobek naukowy na potrzeby wykonywanego zawodu.

Przygotowanie merytoryczne absolwenta geografii turyzmu Uniwersytetu Łódzkiego umożliwia mu podjęcie pracy administratora i organizatora turystyki i wypoczynku w zakładach pracy (pracownicy służb socjalnych ds. turystyki) oraz $\mathrm{w}$ or . ganizacjach społecznych o różnym profilu działalności. Posiada on również przygotowanie do pracy $w$ biurach turystycznych i wypoczynkowych, gdzie powinien być wykorzystany przy programowaniu i organizacji zarówno imprez turystycznych, jak również czynnego wypoczynku. Absolwenci specjalizacji kończąc studia 
uniwersyteckie są również przygotowani do pracy z młodzieżą, zarówno w szkole, jak i innych placówkach i organizacjach oświatowych. Mogą oni równolegle z pracą pedagogiczną stać się organizatorami ruchu krajoznawczego w różnych jego for. mach i zakresach.

W artykule przedstawiono również tematykę prac magisterskich podejmowanych przez studentów tej specjalizacji oraz podano wykaz głównych podręczników i skryptów zalecanych studentom geografii turyzmu na Uniwersytecie Łódzkim.

\section{SUMMARY}

Education of specialists for the tourism sector is associated at The University of Łódź with the Faculty of Geography, where the Postdiploma Centre of Tourism was opened in 1977, while since 1982 this Faculty has been offering the academic specialization in the geography of tourism.

The concept of educating a geographer of tourism at The Uniwersity of Eodz assumes the training of such 'a graduate who will be equipped with skills of giving content, mainly cognitive content, to various forms of recreation and tourism. He should be an aware organizer of leisure time as regards its active, tourist-cognitive utilization.

The programme assumptions in the specialization of geography of tourism are realized during the third, fourth and fifth study years and they encompass 870 hours of lectures, seminars and classes at the University and 63 days of professional traineeship and field classes (Table I)

The specialization curriculum may be divided into five subject blocks (Table II) The time allotted to various forms of teaching in particular blocks tends to differ, with the main emphasis being placed on a desirable character of education of a future graduate completing university studies. Similarly, the shares of particular blocks in university and off-university activities differ as well.

The present teaching programme assumes that a graduate should:

A - know both physical and psychic capacities and needs of a man wishing to practise tourism;

B - know Poland's tourist attractions and resources, as well as those of the world to be able to propose (in a responsible manner forms and places of practising tourism for a definite man;

C - know how to organize a tourist event and fill up a tourist's free time:

D - be able to use scientific output in a practical way according to needs posed by professional career.

The theoretical and practical education of a graduate of the geography of tourism specialization at The University of Łódź allows them to take positions of organizers and administrators of tourism and resreation in work establishments (employees of socio-welfare services in charge of tourism) and in social organizations with different activity profiles. They are also prepared to work in tourist agencies, where they should be employed in work connected with the pragramming and organizing of both tourist events and active recreation. The graduates of this specialization are also prepared to work with young people both schools and in other educational institutions and organizations, where alongside performing teaching jobs they may become organizers of tourist activities in different forms. 